\title{
Extra plasticity governed by shear band deflection in gradient metallic glasses
}

\section{Yao Tang ( $\nabla$ tangyao@zju.edu.cn )}

International Center for New-Structured Materials (ICNSM), Zhejiang University

\section{Haofei Zhou}

Zhejiang University https://orcid.org/0000-0001-9226-9530

\section{Xiaodong Wang}

Zhejiang University

\section{Qingping Cao}

Zhejiang University

\section{Dongxian Zhang}

Zhejiang University

\section{Wei Yang}

Zhejiang University

Jian-Zhong Jiang

Zhejiang University

\section{Article}

Keywords: Controlled Structural Gradients, Heat Treatment Engineering Protocol, Cryogenic Thermal Cycling

Posted Date: April 5th, 2021

DOI: https://doi.org/10.21203/rs.3.rs-366951/v1

License: (c) (i) This work is licensed under a Creative Commons Attribution 4.0 International License. Read Full License

Version of Record: A version of this preprint was published at Nature Communications on April 19th, 2022. See the published version at https://doi.org/10.1038/s41467-022-29821-4. 


\title{
Extra plasticity governed by shear band deflection in gradient metallic glasses
}

\begin{abstract}
Inspired by gradient materials in nature, advanced engineering components with controlled structural gradients have attracted significant research interest due to their exceptional combinations of properties. However, it remains challenging to generate structural gradients that penetrate through bulk materials, which is essential for achieving enhanced mechanical properties in metallic materials. Here, we propose a heat treatment engineering protocol to realize a controllable structural gradient in bulk metallic glasses (BMGs). By adjusting the holding time of cryogenic thermal cycling, a series of BMGs with gradient-distributed free volume contents from internal to external can be synthesized. Both mechanical testing and atomistic simulations demonstrate that the spatial gradient can endow BMGs with extra plasticity without sacrificing their ultrahigh strength. Such an enhanced mechanical property is governed by the gradient-induced deflection of shear deformation that fundamentally suppresses the unlimited shear localization on a straight plane that would be expected in BMGs without such a gradient.
\end{abstract}


Advances in modern science and technology continue to impose more stringent requirements for engineering materials, including exceptional strength and toughness. Unfortunately, these two properties are almost mutually exclusive in monolithic materials ${ }^{1,2}$. Obtaining optimal mechanical performance is always a compromise, one which can be achieved by optimizing the microstructure through empirical design. Notably, the introduction of structural gradients can overcome the strength-ductility trade-off in metallic materials and give rise to high-performance functionalities ${ }^{3-8}$. Concerning such gradients, nature provides a rich source of inspiration. Many natural materials have highly sophisticated structures with complex gradient designs that possess extremely impressive combinations of properties significantly surpassing those of their constituents ${ }^{9-13}$. In view of the gradient structures of natural materials, exploring structural gradients to enhance the properties of engineering materials has generated strong interest. Typical examples are widely-exploited gradient metals with nanograined $(\mathrm{NG})^{14}$ or nano-twinned (NT) structures. ${ }^{15}$ In contrast to conventional homogeneous coarse-grained (CG) materials, the deformation mechanism of gradient nanostructured (GNS) materials is often heterogeneous and is regulated and constrained by the gradient structure. Also, structural gradients typically cause stress gradients and even activate new dislocation structures ${ }^{8}$. Nevertheless, current GNS materials are limited to a few, pure face-centered-cubic metals and typical alloys. For example, $\mathrm{Mg}$ alloys can be strengthened by introducing a gradient nanograined structure while this strategy is unable to provide large ductility in Mg alloys. Recently, an Mg-based nano dual-phase metallic glass (NDP) coated on a gradient nano-grained Mg alloy showed enhanced ductility and yield strength compared to the base alloy ${ }^{16}$. The success of this design strategy of combining heterogeneous metallic glass (MG) and gradient nanograined structure provides us a motivation to extend the principles of structural gradients to amorphous systems in designing 'intrinsic' gradient MGs (GMGs). Indeed, MGs with extraordinary physical and biomaterial properties have recently been developed ${ }^{17}$, but severe brittleness holds one major weakness that precludes the wide application of MGs. The introduction of spatial gradients may offer a promising solution for tuning deformation behavior and enhancing the plasticity of MGs.

Over the past several years, various fabrication methods have been applied to develop structural gradients in engineering materials. The fabrication methods can be divided into two categories: bottom-up methods, including physical and chemical deposition $^{18}$, layer-by-layer assembly ${ }^{19}$, and three-dimensional printing ${ }^{20}$; and top- 
down methods including surface mechanical treatment methods ${ }^{21-23}$, laser shock peening ${ }^{24}$, and roll bonding ${ }^{25}$. Despite their widespread use in engineering design, these methods suffer from marked constraints. Bottom-up methods are generally only feasible for making thin films or microscopic samples. Existing top-down methods, on the other hand, have limits for the range of bulk gradient materials. For instance, surface mechanical treatments always produce a limited volume fraction of gradients only near the surface, or they generate a negligible degree of structural gradients along the gradient direction. All of the aforementioned issues limit our ability to achieve a gradient throughout the bulk metallic glass (BMG) samples. It is essential to develop new strategies and practical methods to design and fabricate gradient BMGs to tailor their mechanical properties.

In this paper, we report a design strategy and a set of simple fabrication methods to produce the GMG in bulk form by introducing a controllable spatial gradient of the free volume content. Through experiments and MD simulations, we demonstrate that the excellent performance of GMG can be attributed to its "shear band deflection" capability that arises from its intrinsic gradient structure. A notable difference in the local free volume defects the angle of the shear band initiation and propagation. Using model heterogeneous materials, we discuss the atomic-scale origin of the observed variations in the SB dynamics and the angle with changing structural state. The design strategy offers a simple and yet versatile method to improve the mechanical properties of BMGs and, more importantly, to design new generations of high-performance structural materials.

\section{Results}

The design strategy for gradient BMG. The design strategy for the GMG structure is proposed in Fig. 1. The plastic deformation of uniform BMGs is through shear localization into narrow bands (Fig. 1a). Such localization often leads to the running away of one dominant shear band, eventually leading to catastrophic failure and macroscopic brittle behavior ${ }^{26}$. The shear band plane in BMGs occurs along an angle at which the corresponding effective shear stress is maximized, which suggests the important influence of normal stress on the shear plane ${ }^{27,28}$. The normal stress effect on deformation in MG lies in the principle of atomistic friction, as embodied in the MohrCoulomb criterion: 


$$
\tau_{y}=\tau_{0}-\alpha \sigma_{n}
$$

where, $\tau_{y}$ is the effective shear yield stress, $\tau_{0}$ is a constant, and $\alpha$ is an effective coefficient of friction that controls the strength of the normal stress effect ${ }^{29,30}$.

We propose that the plasticity of BMGs can be enhanced through a gradient design of the microstructure, with the free volume concentration increasing from the outer to the inner part of the cylindrical BMG specimen (Fig. 1b). Changes in the free volume content and bonding conditions of structural units are expected to modify friction coefficient $\alpha$ and therefore shear band angle $\theta$. Figure 1c shows schematic illustrations of the development of shear bands in such a gradient BMG specimen. The primary shear band initiates at the upper-left surface with a relatively lower content of free volumes, corresponding to a relatively larger friction coefficient $\alpha$. Taking normal stress into consideration, the effective shear yield stress is maximized at shear band angle $\theta$ for the local hard region. As the shear band progresses toward the central soft region of the specimen, the increasing value of free volume concentration alters the normal stress effect on the shear band, inducing a gradual increase in the shear band angle. The shear band is thus deflected by the structural gradient of the BMG. Afterwards, as the shear band propagates from the center to the lower-right surface, free volume concentration declines, leading to a gradual decrease in the shear band angle and a reversed deflection pattern of the shear band. In short, the deflected shear band path avoids the, otherwise straight, transecting shear band across the whole sample, which provides a promising route for improving the plastic deformability of BMGs.

Characteristics of the GMG. To test this design strategy, we selected a relatively brittle $\mathrm{Zr}_{58} \mathrm{Cu}_{22} \mathrm{Fe}_{8} \mathrm{Al}_{12} \mathrm{MG}$ to construct the gradient $\mathrm{BMG}$ structure. We used cryogenic thermal cycling between a certain temperature (323 K) and cryogenic temperature (77 K) to prepare the gradient samples. Figure 2a shows a detailed description of the cryogenic thermal cycling treatment apparatus used to introduce a gradient rejuvenation into the cylindrical MG samples. When the sample was first heated at a certain temperature and then cooled to a low temperature, the rejuvenation behavior came first, which is mainly related to the quasi-localized vibrations of atoms in the flow unit surrounded by the elastic matrix. The sample must be held for a sufficiently long time to enhance its rejuvenation effect. With a long holding time at high temperature, the atoms within flow units will move cooperatively and reversibly on a large scale, and 
resulting in a fast relaxation in turn. Therefore, it can be expected that dynamic rejuvenation or relaxation behaviors vary at internal and external parts of the sample during their evolution with time. Controlling the holding time can induce gradient rejuvenation (free volume content) processes.

Figure $2 b$ shows the results of density measurements of the as-cast sample and the treated samples. The treated samples refer to ten-cycled samples using the same thermal cycling process but with different holding times, as shown in Fig. 2c. The density of the treated samples is lower than that of the as-cast samples, which suggests a relatively large free volume content. The density of the treated sample decreases with the increased holding time but seemingly saturates when $t$ is larger than about $60 \mathrm{~s}$. Figure $2 \mathrm{~d}$ shows the variation of hardness across the diameter on a cross-section of the $2 \mathrm{~mm}$ cylindrical as-cast and treated samples (the top of Fig. 2e shows the method for hardness measurements along various circles, in which 8 indentations were performed to acquire an average harness value at each circle, together with 80 indentations from the center to the edge). For the t6 sample (treated with a $6 \mathrm{~s}$ holding time), the hardness exhibits little decrease from a distance of $0.3 \mathrm{~mm}$ to the center. Notably, a gradient of the hardness value can be detected for the $\mathrm{t} 25$ sample. From the edge to the center, the hardness value of the t25 sample tends to decrease from $500 \mathrm{HV}$ to $485 \mathrm{HV}$, respectively. In particular, a more obvious hardness-value gradient can be seen for the $\mathrm{t} 70$ and $\mathrm{t} 150$ samples. The gradient hardness values suggest that this method can provide a potentially low-cost manufacturing process for the scalable production of GMGs.

Transmission electron microscopy (TEM) analyses were performed to characterize the amorphous structure at different positions of the 150 GMG sample (bottom of Fig. 2e). Specifically, Figure 2f-h display the TEM images of edge, middle, and center regions (labeled A, B, and C) for the t150 sample. One can clearly see a grain-like microstructure with a dark-bright contrast in the sample at the edge. Of significant interest, for the sample in the middle, the sizes of the dark and bright regions are enlarged to $2-3 \mathrm{~nm}$. The characteristic length of the inhomogeneous microstructure reaches $5 \mathrm{~nm}$ in the sample at the center. Considering that our TEM results show an increasing heterogeneity with a decreasing distance from the center, the hypothesis suggested by Ketov et al. provides a reasonable explanation for the gradient amorphous microstructures $^{31}$. Ketov et al. attribute the rejuvenation effects to intrinsic nonuniformity of the glass structure, which gives a non-uniform coefficient of thermal expansion. The population and intensity of soft spots (dark), with lower elastic stiffness 
and higher CTE, increases with cycling. Hence, the brighter contrast in our TEM images at the center region may result from a lower density zone, while the edge region has a relatively high density in our treated samples. Following the corresponding selected area electron diffraction (SAED) patterns (insets of Fig. 2f-h), we confirm that the observed microstructures are all structurally amorphous. The radial distribution functions (RDFs, Fig. 2i), which were calculated from the SAED patterns of three parts in the 1150 sample, have differences in their peak positions. It was found that the first peak position in RDFs is shifted to higher $r$ values from the edge to the center of the treated sample, i.e., the average atomic bond distances increased from edge to center. All observed experimental results - i.e., at the center with a reduction in hardness, larger bright contrast in TEM images, and larger average atomic bond distance - reveal an enhancement of free volume in the center part. Therefore, gradient structure (or rejuvenation) indeed occurs from edge to center in the treated samples.

Mechanical behavior and deformation mechanisms of GMG. To demonstrate the enhanced plastic deformability of gradient BMGs, we compared the engineering compressive stress-strain curves of the gradient BMG samples with the as-cast sample (Fig. 3a). The as-cast sample undergoes negligible plastic strain before fracture, typical of the strong-and-brittle behavior reported in the literature. Of importance, plasticity strongly increased significantly without the expense to the strength in the gradient samples, reaching maximum when the holding time was $70 \mathrm{~s}$ and $150 \mathrm{~s}$. For comparison, Fig. $3 \mathrm{~b}$ summarizes variations of measured plastic strain as a function of the structural gradient. The structural gradient is a parameter that quantifies the structure difference of cryogenically treated samples as the change in hardness per unit thickness along the gradient direction. As illustrated in Fig. 3b, we observed an increase in plastic strain with the increase of the structural gradient. The samples with a structural gradient of 16 $\mathrm{HV} / \mathrm{mm}$ exhibited the largest plastic strain, about four times that of the as-cast sample. The above observations strongly suggest that a substantial plasticity increment can be achieved solely by introducing the structural gradient.

To uncover the physical mechanisms underlying ductility enhancement in the GMGs, we explored the key structural parameters that are affected by the structural gradient. Figure 3c-g display the lateral morphologies of the as-cast and GMG samples obtained by SEM after the final failure. One dominating primary shear band plane, a typical failure mode observed in brittle MGs, exists in the as-cast sample (Fig. 3c). In 
contrast, the GMG samples demonstrate interesting fracture surface morphology. For the t6 sample (Fig. 3d), the fracture surface looks somewhat uneven. A small bulge on the fracture surface can be seen for the $\mathrm{t} 25$ sample. For the $\mathrm{t} 70$ and $\mathrm{t} 150$ samples (Fig. $3 \mathrm{f}$ and $\mathrm{g}$ ), some humps can be clearly observed in the center region of the fracture surface. These fracture surfaces in Fig. 3c-g indicate that the shear band plane deflects obviously from the original shear planes when the shear bands propagate into the center of the GMG samples. To accurately characterize these fracture surfaces, threedimensional (3-D) profiles of the fracture surfaces are also displayed in Fig. 3h-1. Ridges running parallel to the shear band plane direction in the middle (see line in Fig. $3 \mathrm{~h}$ ) are connected by a set of transverse ridges. It can be seen that the ridges in the fracture surface of the as-cast and t6 samples are almost flat. For the $t 25$ sample, the branching of the ridges as well as their meandering in different directions as the shear plane front advances can be noted. An obvious hump can be observed in the height variation along the shear band plane from top to bottom of the fracture surfaces in the t70 and t150 samples. The shear band plane looks like a non-uniform surface with a bent shape, which means that the fracture angle changes at a particular stage during shear band propagation. Distributions of ridge heights, obtained from 2-D profiles of fracture surfaces along the dash line (Fig. 3h), are shown in Fig. 3m. The relative homogeneous as-cast sample only shows small height fluctuation. In contrast, the GMG samples exhibit marked height differences, i.e. lower on both sides and higher in the middle. From the calculated fracture angle at each point along this variation line, the fracture angle in the center is larger than that in the outer part, which suggests that shear band deflection indeed occurs during deformation. It is apparent that this is direct evidence that a novel non-uniform deformation mechanism in GMG occurred. The structural gradient exerts a direct influence on the shear banding, and the deflection of the shear band is thought to be closely related to the variation of the free volume content. In the edge region near the fracture origin of samples, a larger $\alpha$ value might be expected, and consequently a larger effect of normal stress. In this case, the more difficult is the expected movement among structural units. The material also shows brittle behavior - hence the small angle of the shear band plane. On the other hand, a smaller $\alpha$ might be associated with an increase in free volume content by gradient rejuvenation in the center part, leading to easier plastic flow and a larger angle of shear band plane. Our SEM images of the fracture surface morphology verify the abovementioned analyses. Fig. 3n-s clearly show the morphologies of fracture as-cast and 
t150 samples at different positions. The three positions of the as-cast sample exhibit typical viscous, river-like patterns along the shear direction with very narrow spacing. Distinct surface morphologies developed in the center regions of the two samples, which indicates a difference in the local free volume content. A clear vein pattern was observed in the center of typical t150 BMGs (position 5) in Fig. 3r. All these fracture morphologies reveal a novel deformation mechanism related to the controlled variation of free volume content in GMGs.

Atomistic mechanism of shear band deflection. Beyond these encouraging experimental results, we examined atomic-level details of the observed shear band deflection process in GMGs. Microscopically, the shear-banding process is controlled by shear transformation zone (STZ) percolation ${ }^{32}$. Owing to spatial and temporal confinement, detailed characterization of STZs in GMGs is impossible to probe experimentally. The molecular dynamic simulation method offers a powerful approach to explore the fundamental characteristics of shear band deflection and derive an atomistic description of the deflection mechanism. A typical gradient $\mathrm{Cu}_{65} \mathrm{Zr}_{35} \mathrm{MG}$ consisting of two regions with disparate amounts of free volume is created by randomly removing $2 \%$ of atoms in the right half of the simulated MG box - as shown in the inset in Fig. 4a, the right region (blue) of the gradient model is soft. Here, we selected $\mathrm{Cu}-\mathrm{Zr}$ system as a prototype because of its high quality of potential, which has been well developed and frequently applied ${ }^{33}$, while no potential is available for $\mathrm{Zr}_{58} \mathrm{Cu}_{22} \mathrm{Fe}_{8} \mathrm{Al}_{12}$ MG. Figure 4a depicts the variation of atomic volume along the Y-direction in the ascast and gradient MG models. As-cast MG has almost no free volume fluctuation due to its relatively homogeneous structure, whereas GMG displays an obvious larger atomic volume value in the right part. Figure $4 \mathrm{~b}$ depicts the calculated compressive stress-strain curves of the as-cast and gradient MGs. The calculated stress-strain curve of the relatively uniform as-cast MG with less free volume exhibits a yield strength and a distinct stress drop after about 7\% strain, indicating substantial shear localization. In contrast, the stress drop is much less pronounced in the GMG. More interestingly, the GMG exhibits enhanced average flow stress in the 10\%-15\% strain region relative to the as-cast MG, which indicates enhanced plasticity in the GMG. This can be also deduced from the plastic deformation of as-cast and gradient MGs at $9 \%$ strain. As shown in Fig. 4c, the configurations of the shear bands observed in a GMG are very different from those in a relative uniform as-cast MG. For the as-cast MG, the shear 
band almost penetrated the entire sample along the maximum shear plane with a rough straight line. In contrast, the propagation appeared to be changed when it penetrated the gradient-transition region in the GMG. It is apparent that the shear band angle becomes significantly larger in the right soft region due to the presence of a large free volume and a small friction coefficient. These results, obtained by simulations, suggest that gradient free volume content plays a vital role in deflecting the propagation of shear bands in the region with changed Mises strain distribution, ultimately giving rise to the improved plasticity of the glass, which is roughly consistent with the experimental observations.

What is more, the shear band is not only related to the percolation of STZs, it is also related to the consecutive activation of STZs based on successive strong strain

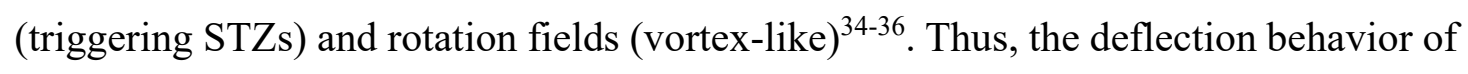
shear banding can be greatly affected by non-uniform stress/strain fields. Variation of the free volume content across the gradient-transition region not only perturbs the strain distribution but also changes the rotation fields, which can be obtained by analyzing the rotational part of the deformation gradient tensor. As shown in Figure 4d, for the ascast MG, two rotation fields around the STZ can be clearly identified. The direction of rotation is clockwise (white color), along with the shear front, and anti-clockwise (black color) if one moves perpendicular to it. More specifically, the clockwise rotation fields are strongly connected and concentrated in one shear band, whereas the anti-clockwise rotation fields are nearly uniformly distributed throughout the whole sample. These observations are consistent with the STZ-vortex mechanism ${ }^{34}$ proposed by Sopu et al. They reported that STZs can induce collective vortex-like motions in the shear front. The vortex-like motions in turn act as a medium, triggering the activation of successive STZs, and finally cause the rapid propagation of the shear band. For the GMG, the rotation fields around the STZ in the left part are similar to those observed in the ascast MG. However, the rotation fields around STZs clearly change in the right soft region. STZ percolation follows a new specific direction with a larger angle. Previously accumulated clockwise rotation fields are discontinuous within the single shear band. Meanwhile, new clockwise STZ-vortex sequences are activated with a deviation from the maximum shear band plane. The anti-clockwise rotation fields become weaker and decrease around the main shear band. To better visualize the change in the rotation fields, the rotation fields across the shear bands at different positions in the GMG are depicted in Fig. 4e, which shows the variation in rotation angle corresponding to lines 
1-3 in Fig. 4d. Due to the accumulation of the clockwise STZ-vortex in the shear band, the symmetric profiles for the uniform as-cast MG (line 1) show a flat section with positive rotation angles. With the increased distance from the center of the shear band, the rotation angle significantly decreases to negative values, which corresponds to the anti-clockwise rotation fields. The anti-clockwise rotation fields obviously weaken in the gradient-transition region (line 2 ). The rotation angle displays only minor negative values away from the central shear band and varies between 6 degrees and 0 degrees. In the soft region (line 3), the rotation angle is almost the same in this line, and the anticlockwise rotation fields almost vanish. The variation of the rotation fields across the shear band indicates that the STZ vortex-like motion mechanism characteristic in the GMG must be perturbed. This is due to the modification of the local strain field around the STZ caused by the gradient structure.

\section{Discussion}

Figure 4f schematically shows the STZ percolation mechanism under compressive strain in GMG. In the left hard region, since the rotation field shows a quadrupolar-like distribution around the STZs when subjected to compressive stress, the compressive strain is oriented along the y-axis, while the tensile strain is oriented along the x-axis. The activated STZ can perturb the surrounding STZ by generating strong, clockwise rotation strain fields. These clockwise rotation strain fields can compress the STZ located at the top right of the vortex while stretching the STZ located at the bottom left of the vortex. Meanwhile, this activated STZ can also be perturbed by the anticlockwise rotation strain fields generated by surrounding STZs. In this state, the anticlockwise rotation strain fields can also drive the movement of the vortex with a special angle. In this way the following STZ vortex will be activated and the shear band will percolate in this direction. When the STZ vortex moves to the soft region, the weakening of the atomic bonding will perturb the strain field around the STZ. The tensile strain fields will become larger, which will govern the vortex to be closer to the tensile strain direction. By the same reasoning, the influence from the anti-clockwise rotation strain fields will become weak. Hence, the angle of the STZ percolation path changes to a larger value than that in the hard region. The non-activated STZ will be aligned with the new local strain fields and the shear band will percolate in alignment with this new angle - ultimately, the shear band deflected as we observed experimentally. Hence, our model demonstrates that local strain fields can be effectively tuned by the 
free volume gradient, and thus the STZ-vortex motion and shear banding behavior can be controlled. It is still desirable to optimize the gradient structure and strain fields to more effectively control the propagation of shear bands.

In summary, we have proposed a novel gradient BMG design strategy through cyclic heat treatment. The resulting substantial bulk scale structural gradient allows for extra plastic strain without sacrificing ultrahigh strength. Both experimental and computational evidence has demonstrated the importance of covering the whole structure with a tunable free volume gradient for the deflection of shear deformation. We have shown that the deflection of the shear band is accompanied by a gradient change in the vortex motion of the shear transformation zone, which is regulated by the variation of the non-uniform strain fields in the GMG. Our research highlights the potential of creating gradient structured engineering materials with high strength and plastic deformability.

\section{Methods:}

Materials preparation. MGs with the atomic components of $\mathrm{Zr}_{58} \mathrm{Cu}_{22} \mathrm{Fe}_{8} \mathrm{Al}_{12}$ were prepared by arc melting of a mixture of pure elements (99.9\% purity) in an argon atmosphere and injection casting into a copper moldwith a diameter of $2 \mathrm{~mm}$. For cryogenic thermal cycling, one cycle consisted of dipping the sample into liquid nitrogen for a certain period of time $(6 \mathrm{~s}, 25 \mathrm{~s}, 70 \mathrm{~s}$, and $150 \mathrm{~s})$, followed by transferring it into hot water $(T=323 \mathrm{~K})$ for the same time $(6 \mathrm{~s}, 25 \mathrm{~s}, 70 \mathrm{~s}$, and $150 \mathrm{~s})$. All the samples were treated for 10 cycles.

Characterization and mechanical testing. Density measurements, based on the Archimedes method, were conducted using a high precision balance with an accuracy of $\pm 0.01 \mathrm{mg}$. They were repeated at least 15 times to ensure data reliability. Vickers microhardness was measured on the cross-section using a Matsuzawa MMT-X indentation machine with a load of $1.96 \mathrm{~N}$ and holding for $15 \mathrm{~s}$. The microstructure of the samples was examined by TEM using a Cs-corrected FEI Titan G2 80-200 ChemiSTEM. Specimens for TEM were cut from the bulk MG specimens and thinned using a dual-beam FIB system (FEI Helios Nano-Lab 600i). Uniaxial compression tests were performed in an Instron 5982 mechanical testing machine at a strain rate of $1 \times$ $10^{-4} \mathrm{~s}^{-1}$ at room temperature. The specimens, $2 \mathrm{~mm}$ in diameter and $4 \mathrm{~mm}$ in height with two ends, were carefully polished to ensure parallelism. The fracture features and surface morphology of all the samples after compression failure were investigated by 
scanning electron microscopy on a Zeiss Sigma500 field emission gun (FEG) SEM. The height maps of the fracture surface of all the samples were measured by the Dektak XT profile measurement.

Molecular dynamics simulations. Molecular dynamics simulations were performed using a Large-scale Atomic/Molecular Massively Parallel Simulator (LAMMPS) ${ }^{37}$ with newly-developed $\mathrm{Cu}-\mathrm{Zr}$ embedded atom method (EAM) potential ${ }^{38}$. A cubic box containing 2213750 atoms was constructed with three-dimensional (3D) periodic boundary conditions (PBCs) as an initial model. The atoms were randomly arranged, and the system was melted by maintaining a high temperature of $2000 \mathrm{~K}$ for $8 \mathrm{~ns}$ with zero external pressure under the NPT ensemble. The melted model was then quenched at a constant cooling rate of $10^{11} \mathrm{~K} / \mathrm{s}$ from $2000 \mathrm{~K}$ to $50 \mathrm{~K}$ and relaxed at $50 \mathrm{~K}$ for $2 \mathrm{~ns}$ to equilibrate the structure. The final size of the sample was about $120(\mathrm{x}) \times 60(\mathrm{y}) \times 5$ (z) $\mathrm{nm}^{3}$. Afterwards, the gradient MG was prepared by randomly removing $2 \%$ of atoms in the right half of the model (along the Y direction). Once the gradient structure was generated, it was relaxed for 50 ps. The obtained gradient MG was further compressed along the X-direction at $50 \mathrm{~K}$ under a strain rate of $1 \times 10^{8} \mathrm{~s}^{-1}$. The atomic-scale shear banding process was monitored by the atomic shear strain, $\eta^{\text {Mises } 39}$ and rotation field by Ovito $^{40}$.

\section{References:}

[1] Ritchie, R. O. The conflicts between strength and toughness. Nat Mater 10, 817-822 (2011).

[2] Launey, M. E. \& Ritchie, R. O. On the fracture toughness of advanced materials. Adv. Mater. 21, 2103-2110 (2009).

[3] Wu, X. et al. Extraordinary strain hardening by gradient structure. Proc. Natl. Acad. Sci. U.S.A. 111,7197 (2014)

[4] Wei, Y. J. et al. Evading the strength-ductility trade-off dilemma in steel through gradient hierarchical nanotwinsmade pioneering contributions to the mechanics of fracture, dislocations, and stress effects in solid materials. Nat. Commun. 5, 3580 (2014).

[5] Chen, W. et al. Mechanically-induced grain coarsening in gradient nano-grained copper. Acta Mater. 125, 255 (2017).

[6] Zhao, S. T. et al. Generating gradient germanium nanostructures by shock-induced 
amorphization and crystallization. Proc. Natl. Acad. Sci. U.S.A. 114, 37 (2017).

[7] Lu, K. Making strong nanomaterials ductile with gradients. Science 345, 1455 (2014).

[8] Li, X. Y. et al. Mechanical properties and deformation mechanisms of gradient nanostructured metals and alloys. Nat. Rev. Mater. 5, 706 (2020).

[9] Liu, Z. Q. et al. Functional gradients and heterogeneities in biological materials: Design principles, functions, and bioinspired applications. Prog. Mater. Sci. 88, 467 (2017).

[10] Espinosa, H. D. et al. Merger of structure and material in nacre and bone-Perspectives on de novo biomimetic materials. Prog. Mater. Sci. 54, 1059 (2009).

[11] Studart, A. R. Additive manufacturing of biologically-inspired materials. Chem. Soc. Rev. 45, 359 (2016).

[12] Meyers, M. A. et al. Biological materials: Structure and mechanical properties. Prog. Mater. Sci. 53, 1 (2008).

[13] Mayer, G. Rigid biological systems as models for synthetic composites. Science 310, 1144 (2005).

[14] Fang, T. et al. Revealing Extraordinary Intrinsic Tensile Plasticity in Gradient Nano-Grained Copper. Science 331, 1587 (2011).

[15] Cheng, Z. et al. Extra strengthening and work hardening in gradient nanotwinned metals. Science 362, 559 (2018).

[16] Liu, C. et al. Nano-dual-phase metallic glass film enhances strength and ductility of a gradient nanograined magnesium alloy. Adv. Mater. 7, 2001480 (2020).

[17] Zberg, B. et al. MgZnCa glasses without clinically observable hydrogen evolution for biodegradable implants. Nat Mater 8, 887 (2009).

[18] Lin, Y. et al. Mechanical properties and optimal grain size distribution profile of gradient grained nickel. Acta Mater. 153, 279 (2018).

[19] Meng, X. et al. Underwater Mechanically Robust Oil-Repellent Materials: Combining Conflicting Properties Using a Heterostructure. Adv. Mater. 30, 1706634 (2018).

[20] Yang, Y. et al. Electrically assisted 3D printing of nacre-inspired structures with self-sensing capability. Sci. Adv. 5, 9490 (2019).

[21] Li, J. et al. On strain hardening mechanism in gradient nanostructures. Int. J. Plast. 88, 89 (2017). 
[22] Yang, D. K. et al. Work hardening in ultrafine-grained titanium: Multilayering and grading. Acta Mater. 61, 2840 (2013).

[23] Xu, W. et al. Strain-induced microstructure refinement in pure Al below $100 \mathrm{~nm}$ in size. Acta Mater. 152, 138-147 (2018).

[24] Laine, S. J. et al. Microstructural characterisation of metallic shot peened and laser shock peened Ti-6Al-4V. Acta Mater. 123, 350 (2017).

[25] Ma, X. L. et al. Mechanical properties of copper/bronze laminates: Role of interfaces. Acta Mater. 116, 43 (2016).

[26] Greer, A. L. et al. Shear bands in metallic glasses. Mater. Sci. Eng. R. 74, 71 (2013).

[27] Zhang, Z. F. et al. Difference in compressive and tensile fracture mechanisms of $\mathrm{Zr}_{59} \mathrm{Cu}_{20} \mathrm{Al}_{10} \mathrm{Ni}_{8} \mathrm{Ti}_{3}$ bulk metallic glass. Acta Mater. 51, 1167 (2003).

[28] Zhang, Z. F. et al. Fracture mechanisms in bulk metallic glassy materials. Phys. Rev. Lett. 91, $045505(2003)$.

[29] Hsueh, C. -H. et al. Shear fracture of bulk metallic glasses with controlled applied normal stresses. Scr. Mater. 59, 111 (2008).

[30] Baricco, M. et al. Glass formation and mechanical properties of (Cu50Zr50)100-xAlx ( $\mathrm{x}=0,4$, 5, 7) bulk metallic glasses. J. Alloys Compd. 483, 125 (2009).

[31] Ketov, S. V. et al. Rejuvenation of metallic glasses by non-affine thermal strain. Nature 524, $200(2015)$

[32] Schuh, C. A. \& Lund, A. C. Atomistic basis for the plastic yield criterion of metallic glass. Nat. Mater. 2, 449 (2003).

[33] Wu, Z. W. et al. Correlation between structural relaxation and connectivity of icosahedral clusters in CuZr metallic glass-forming liquids. Phys. Rev. B 88, 054202 (2013).

[34] Şopu, D. et al. Atomic-level processes of shear band nucleation in metallic glasses. Phys. Rev. Lett. 119, 195503 (2017).

[35] Şopu, D. et al. Atomic-scale origin of shear band multiplication in heterogeneous metallic glasses. Scr. Mater. 178, 57 (2020).

[36] Scudino, S. \& Şopu, D. Strain distribution across an individual shear band in real and simulated metallic glasses. Nano Lett. 18, 1221 (2018).

[37] Mendelev, M. I. et al. Using atomistic computer simulations to analyze x-ray diffraction data 
from metallic glasses. J. Appl. Phys. 102, 043501 (2007).

[38] Plimpton, S. Fast parallel algorithms for short-range molecular dynamics. J. Comput. Phys. 117, 1 (1995).

[39] Shimizu, F. et al. Theory of shear banding in metallic glasses and molecular dynamics calculations. Mater. Trans. 48, 2923 (2007).

[40] Stukowski, A. Visualization and analysis of atomistic simulation data with OVITO-the Open Visualization Tool. Model. Simulat. Mater. Sci. Eng. 18, 015012 (2010). 


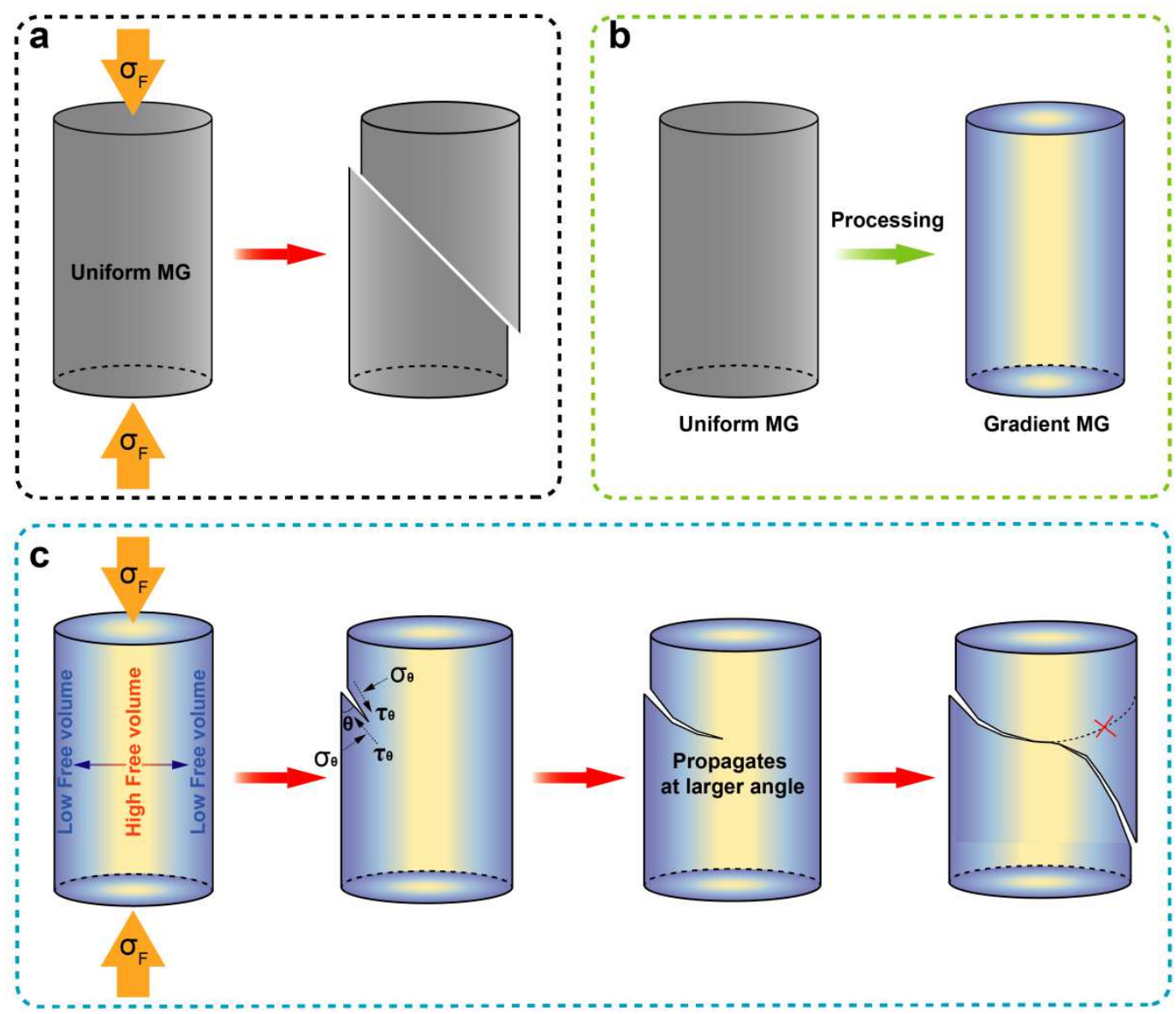

Figure 1. Schematic description of gradient BMG. (a) Schematic representation of one dominant shear band in uniform MG. (b) Schematic diagram of proposed GMG. (c) Proposed shear band deflection mechanism in a GMG during the uniaxial compression process. 

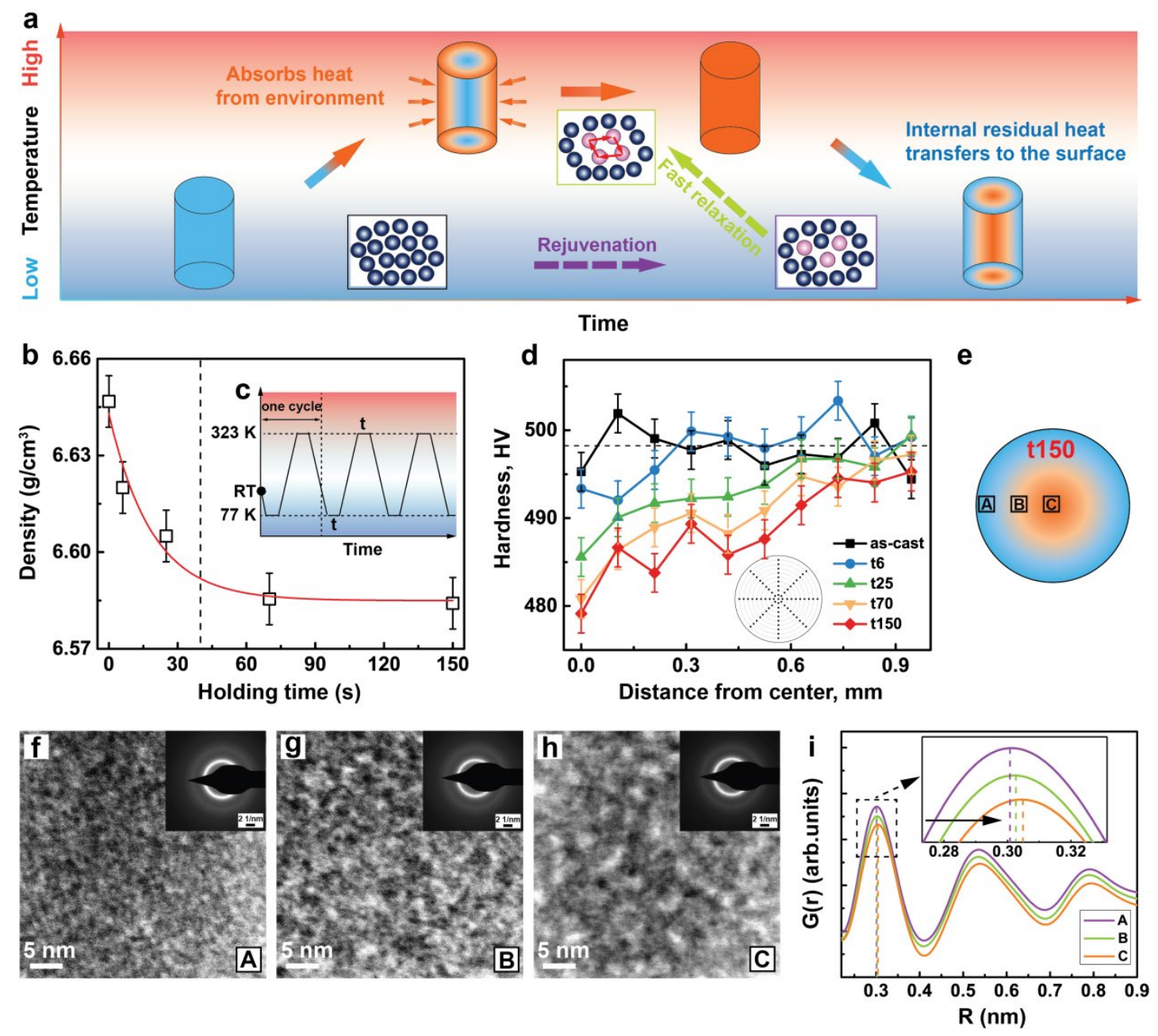

Figure 2. Generation and Characterization of the GMG. (a) Schematic of the GMG by means of CTC. (b) Density values as a function of holding time for the treated MGs. (c) Cryogenic thermal cycling procedure from a high temperature $(323 \mathrm{~K})$ to liquid nitrogen temperature $(77 \mathrm{~K})$, together with the waiting time, $t$, at both the maximum and the minimum temperatures. (d) Variation of average hardness value along the distance from the center. (e) The top shows the method for hardness measurements along various circles. The bottom shows the 150 sample for TEM. (f)-(h) TEM images with the corresponding selected-area-diffraction patterns (SAED) of the edge, middle, and center regions (labeled A, B, C) for the t150 sample. (i) Corresponding radial distribution functions calculated from the SAED of the edge, middle, and center regions (labeled A, B, C) in the $\mathrm{t} 150$ sample. 


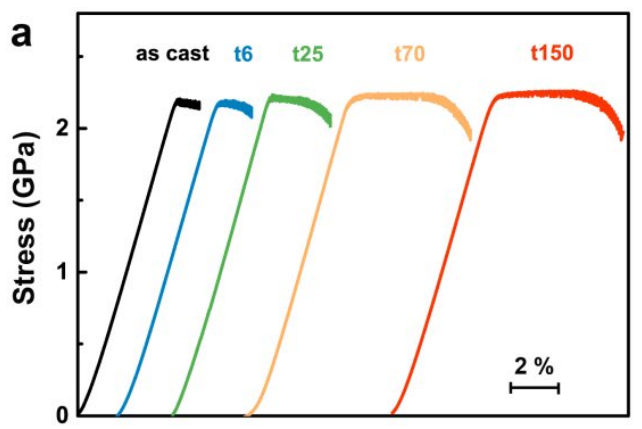

Strain (\%)
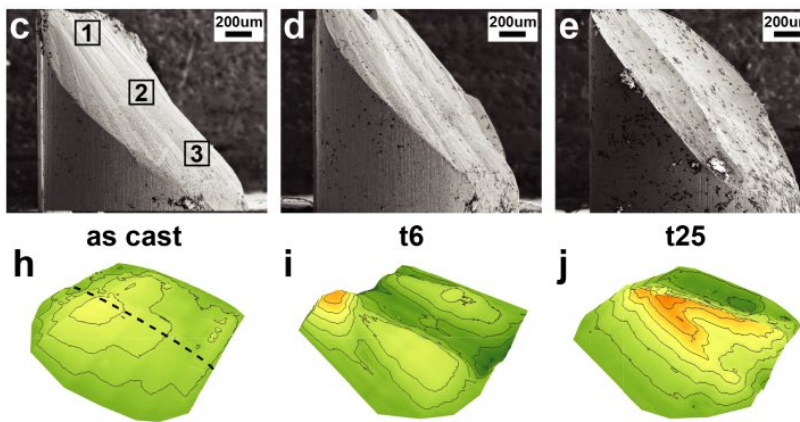

k
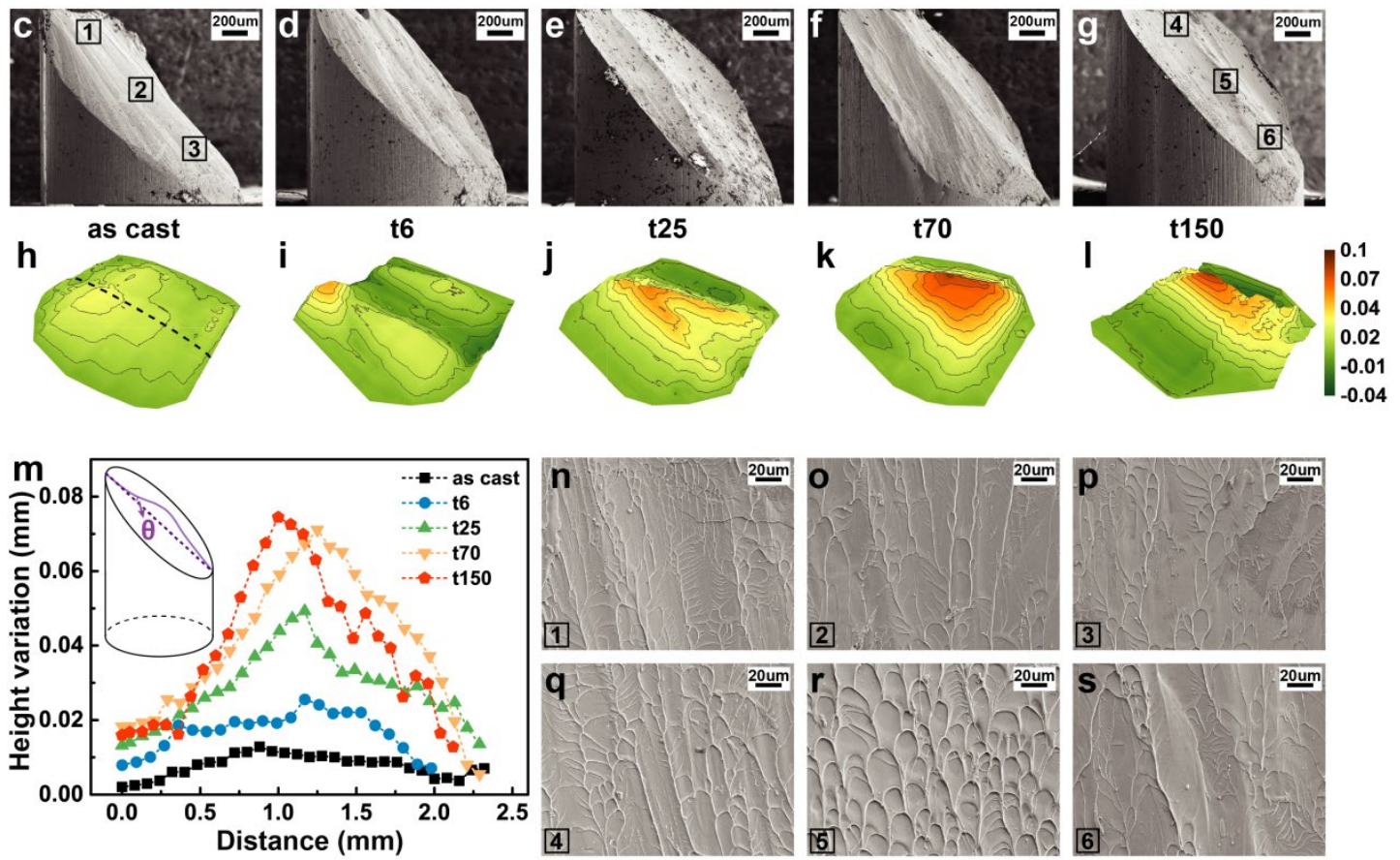

Figure 3. Enhanced mechanical properties, fracture surface change and shear band deflection. (a) Compressive stress-strain curves for the as-cast and treated MGs. (b) Variation of the plastic strain with the structural gradient. (c)-(g) Lateral morphologies of the fractured as-cast and gradient MG samples. (h)-(l) The corresponding 3D contours of the fracture surfaces. (m) Height variation profiles of the middle dash line (h) along the shear band plane for as-cast and gradient MG samples. (n)-(s) SEM surface morphologies at positions 1-3 in the as-cast (c) and 4-6 in the t150 sample (g). 

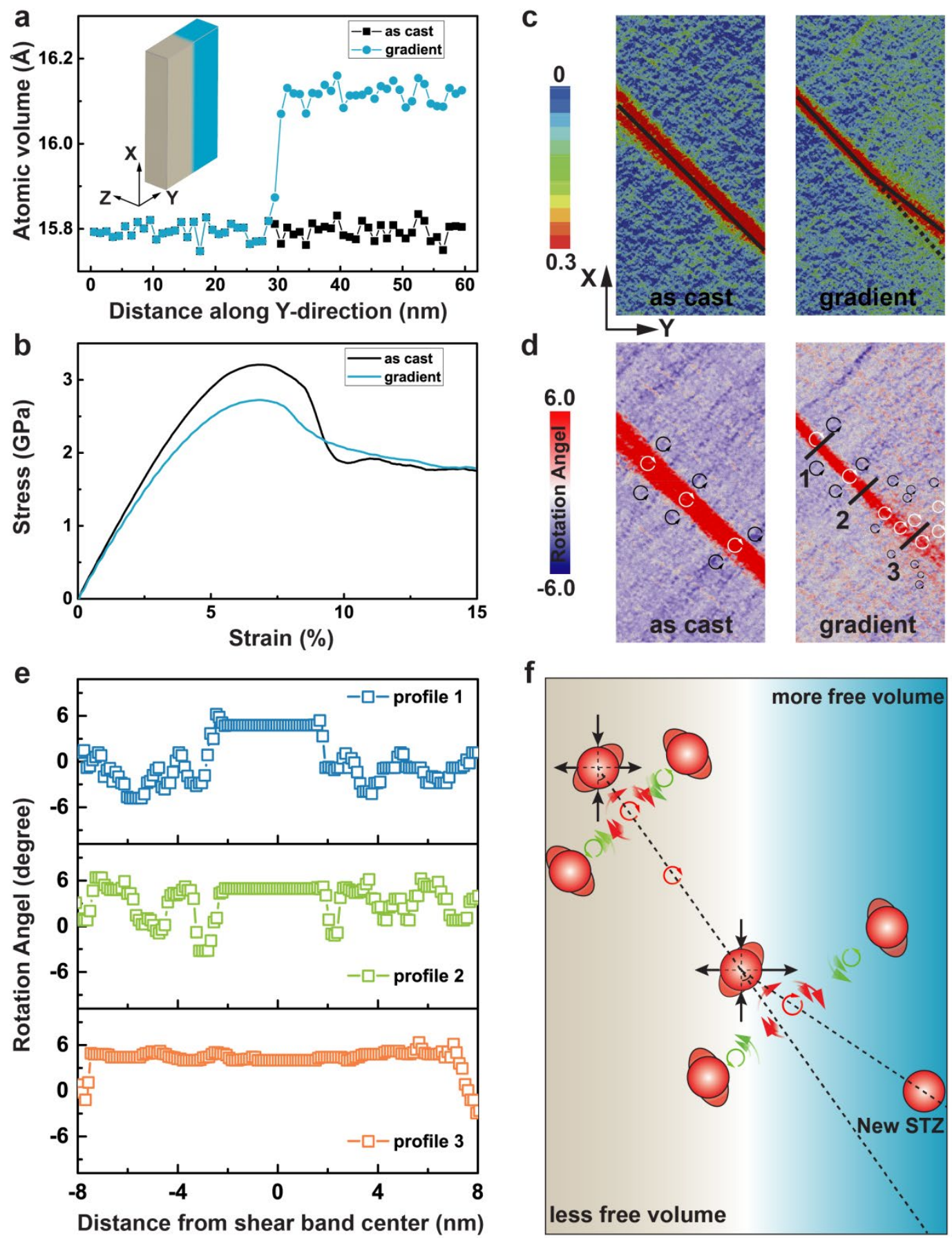

Figure 4. Investigation of the deformation behavior of GMG by MD simulations.

(a) Atomic free volumes as the functions of the position along the Y-direction in as-cast and gradient MGs. Inset shows an illustration of the design of GMG. (b) Representative stress-strain results for the as-cast and gradient MGs during compression along the Xdirection. (c) The spatial distribution of atomic Mises strain (d) Rotation angle of the as-cast and gradient MGs at 9\% compression strain. (e) The variations of the rotation angle along lines 1-3 in (d). (f) Schematic illustration of the STZ percolation mechanism in GMG. 


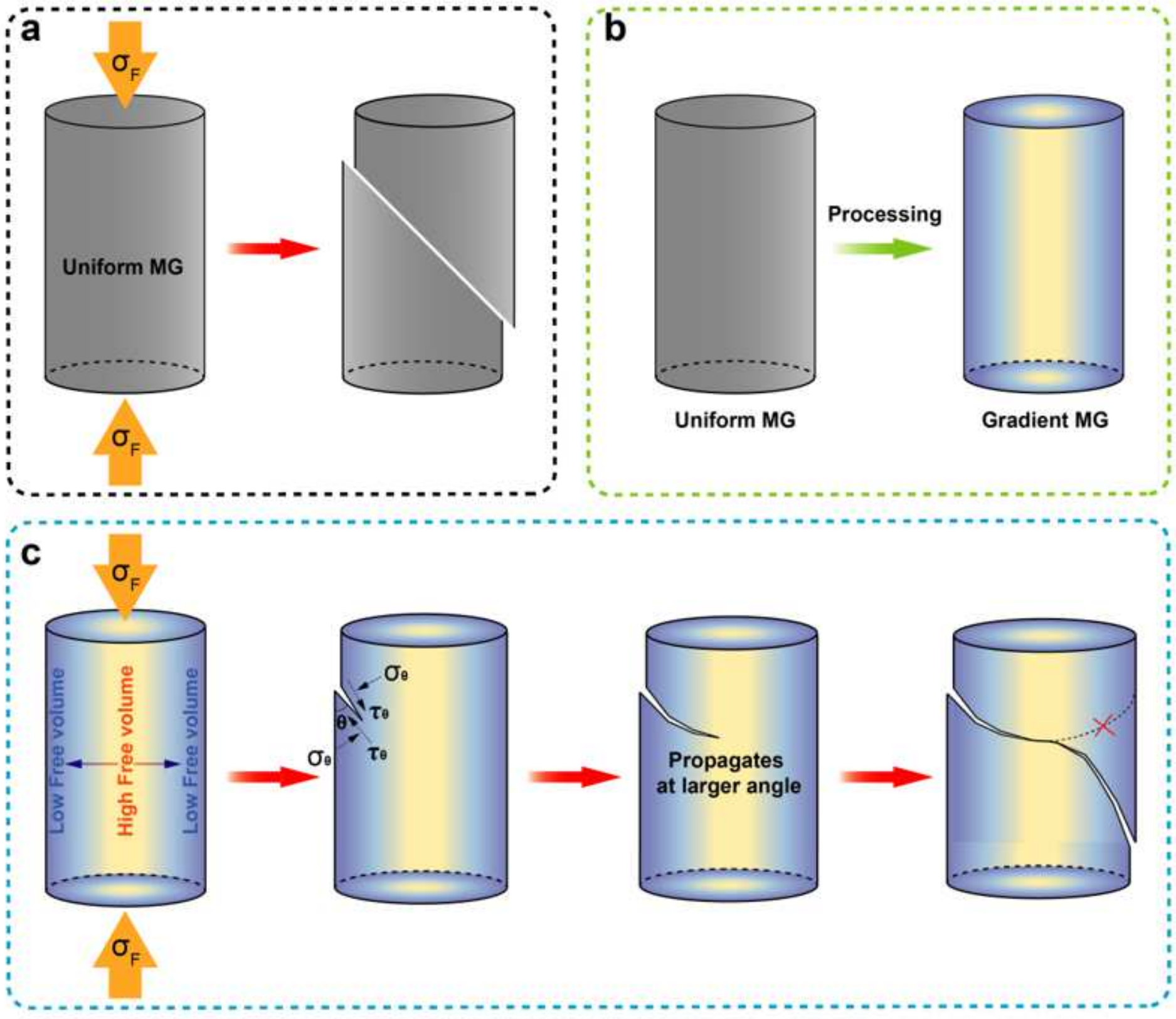

Figure 1

Schematic description of gradient BMG. (a) Schematic representation of one dominant shear band in uniform MG. (b) Schematic diagram of proposed GMG. (c) Proposed shear band deflection mechanism in a GMG during the uniaxial compression process. 


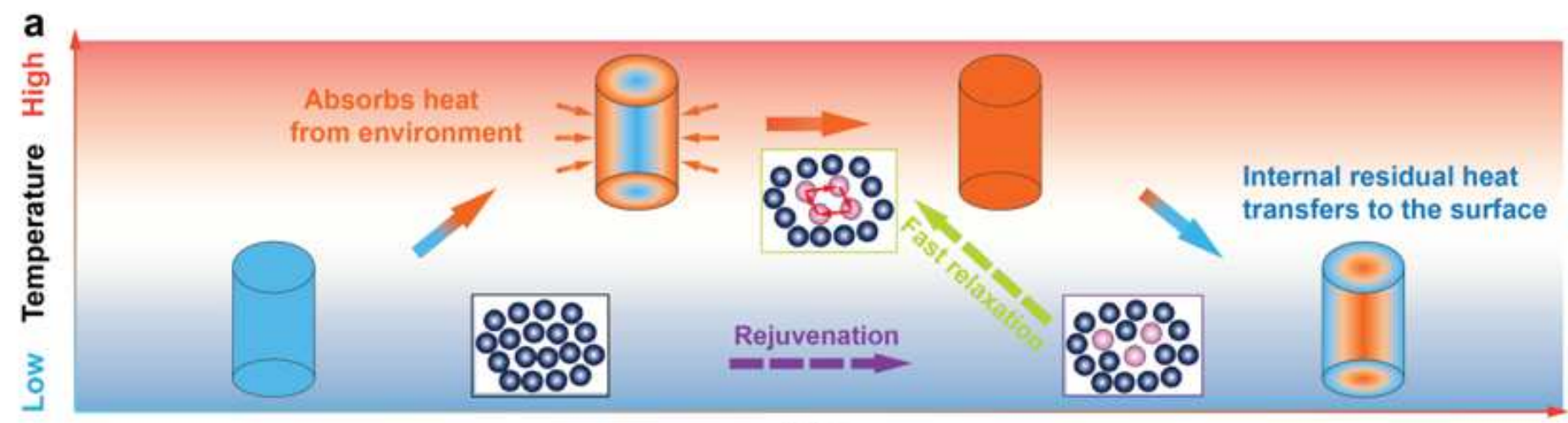

Time
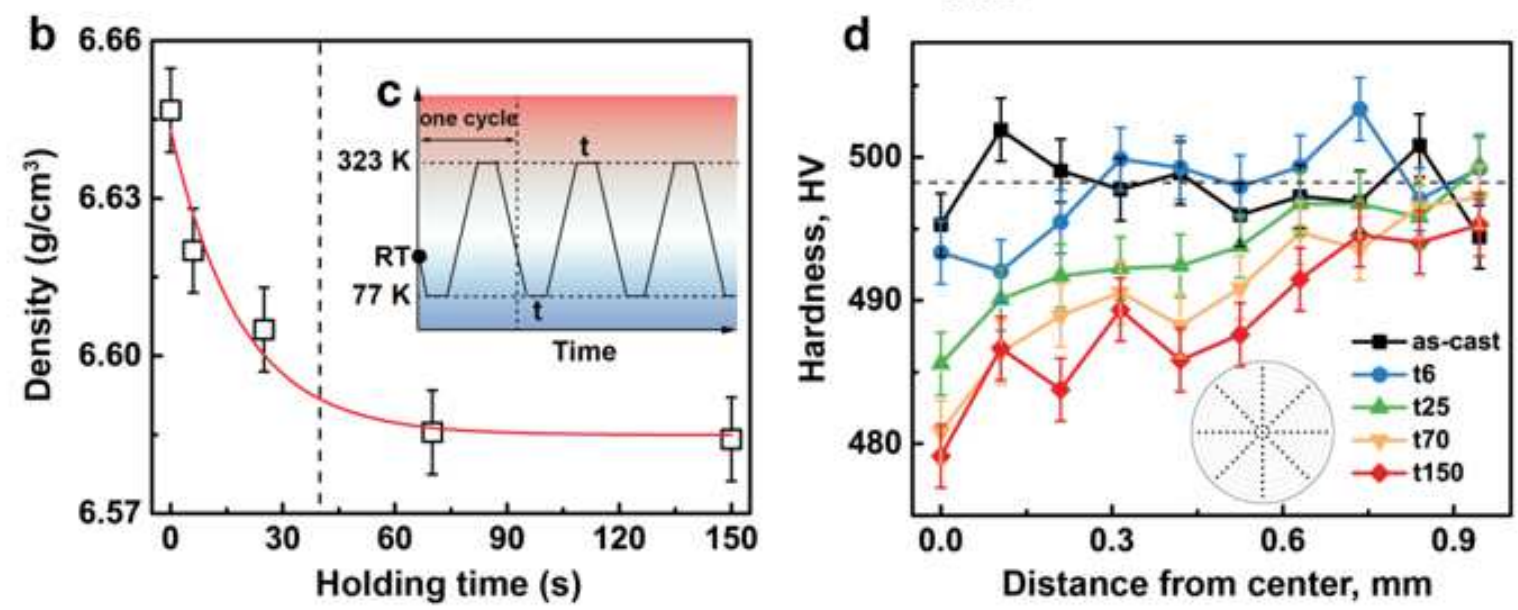

e
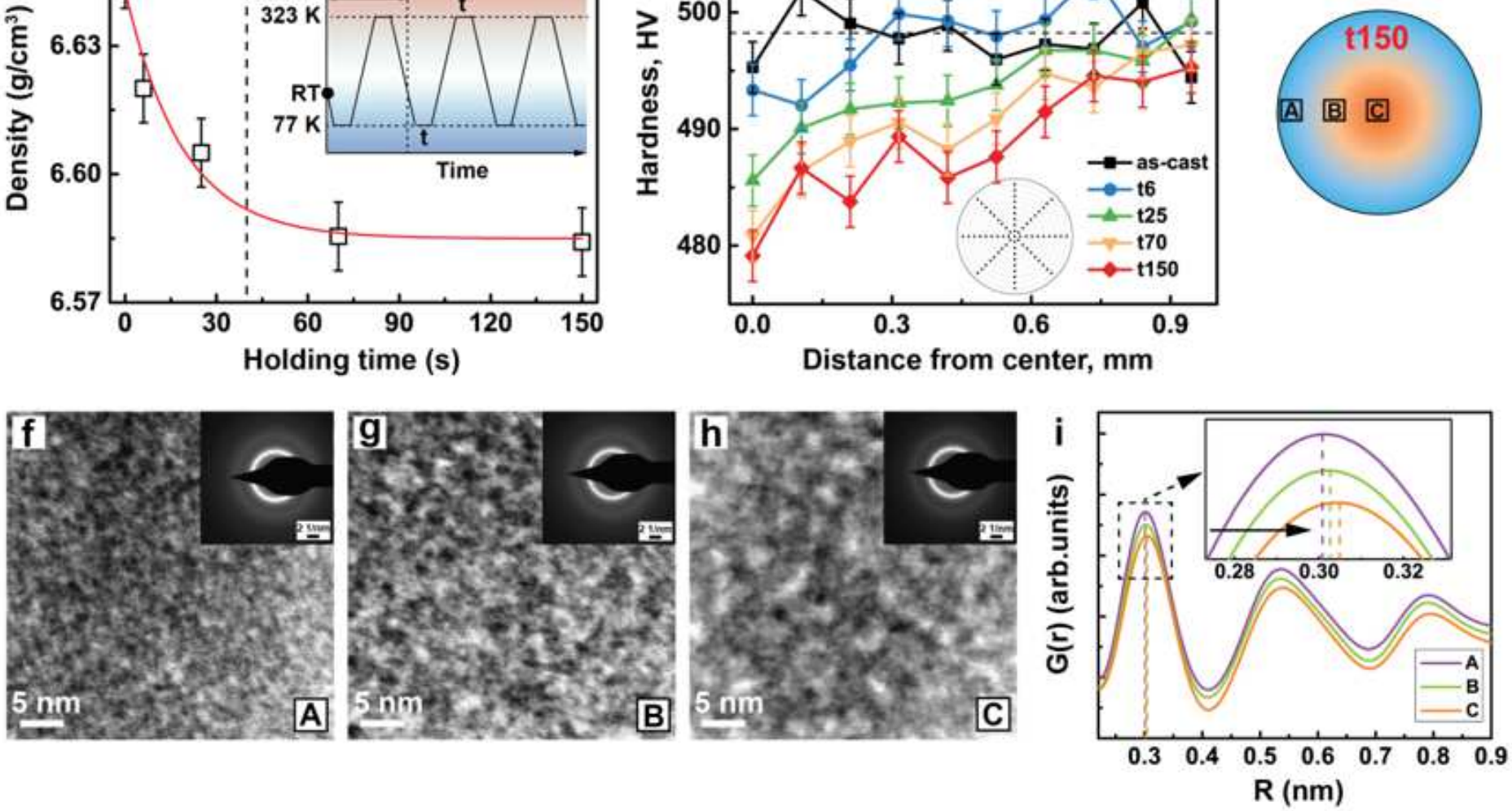

Figure 2

Generation and Characterization of the GMG. (a) Schematic of the GMG by means of CTC. (b) Density values as a function of holding time for the treated MGs. (c) Cryogenic thermal cycling procedure from a high temperature $(323 \mathrm{~K})$ to liquid nitrogen temperature $(77 \mathrm{~K})$, together with the waiting time, t, at both the maximum and the minimum temperatures. (d) Variation of average hardness value along the distance from the center. (e) The top shows the method for hardness measurements along various circles. The bottom shows the t150 sample for TEM. (f)-(h) TEM images with the corresponding selectedarea-diffraction patterns (SAED) of the edge, middle, and center regions (labeled A, B, C) for the t150 sample. (i) Corresponding radial distribution functions calculated from the SAED of the edge, middle, and center regions (labeled $A, B, C$ ) in the t150 sample. 

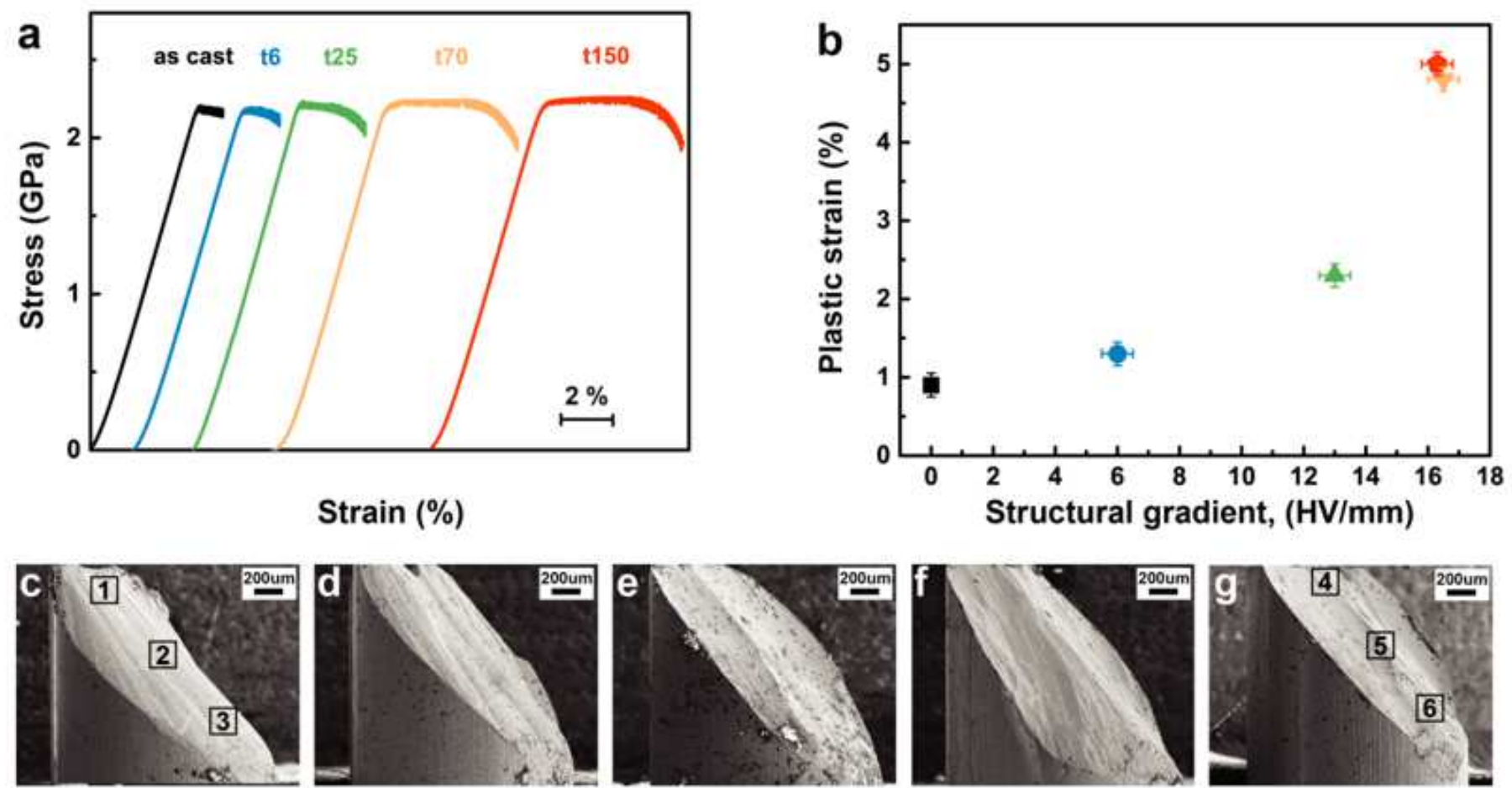

Strain (\%)

Structural gradient, ( $\mathrm{HV} / \mathrm{mm})$
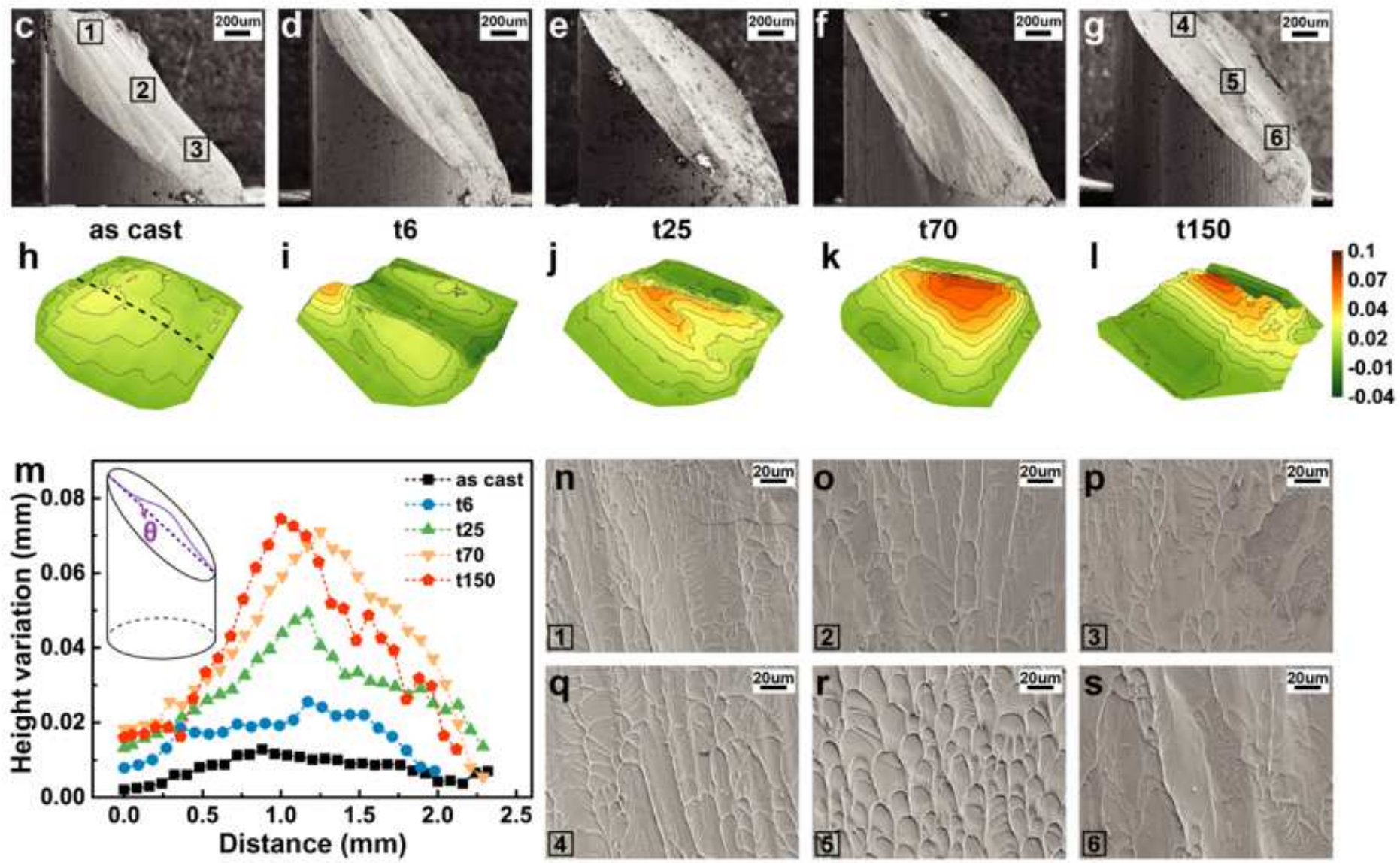

Figure 3

Enhanced mechanical properties, fracture surface change and shear band deflection. (a) Compressive stress-strain curves for the as-cast and treated MGs. (b) Variation of the plastic strain with the structural gradient. (c)-(g) Lateral morphologies of the fractured as-cast and gradient MG samples. (h)-(I) The corresponding 3D contours of the fracture surfaces. $(m)$ Height variation profiles of the middle dash line (h) along the shear band plane for as-cast and gradient MG samples. (n)-(s) SEM surface morphologies at positions 1-3 in the as-cast (c) and 4-6 in the t150 sample (g). 

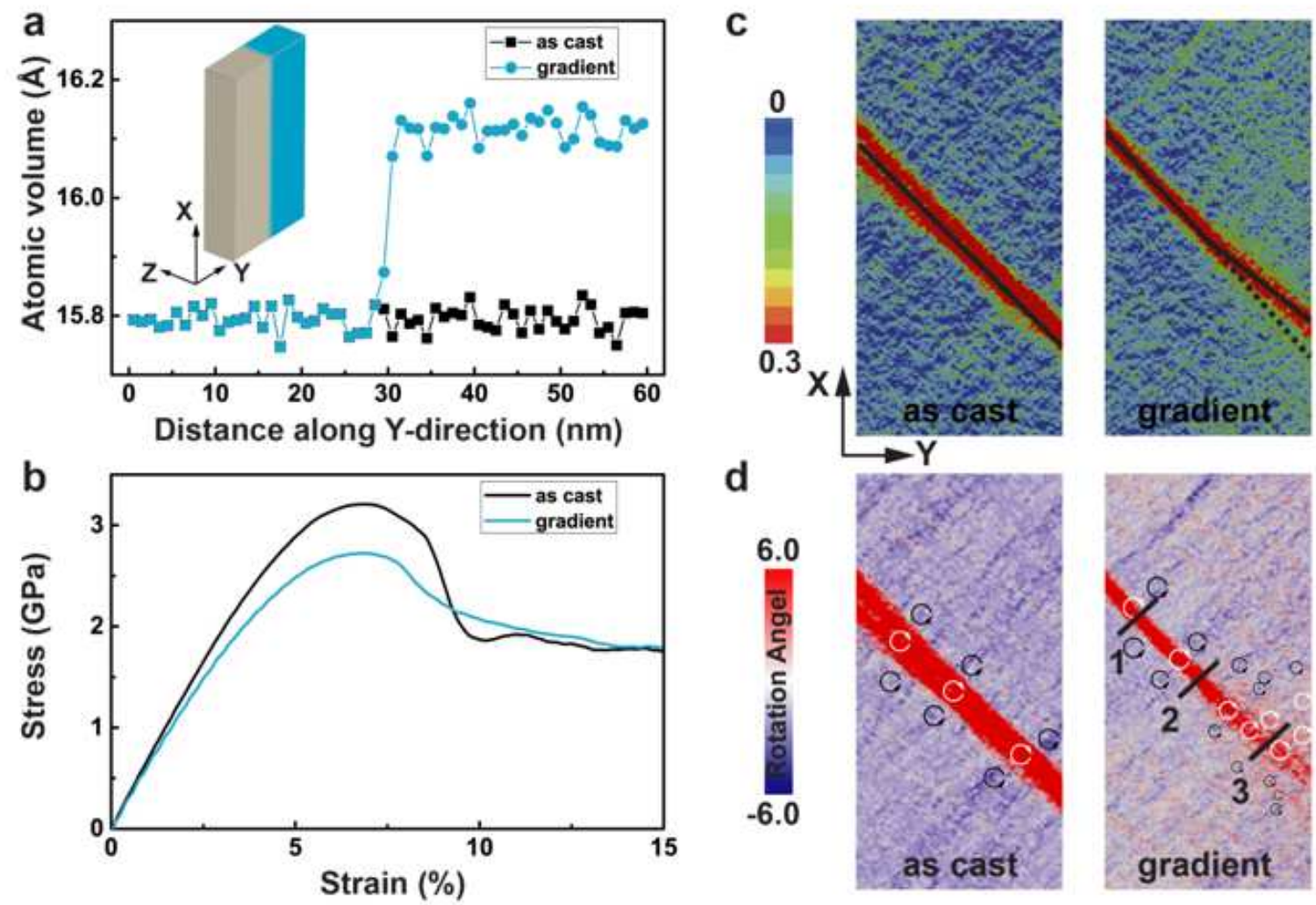

d
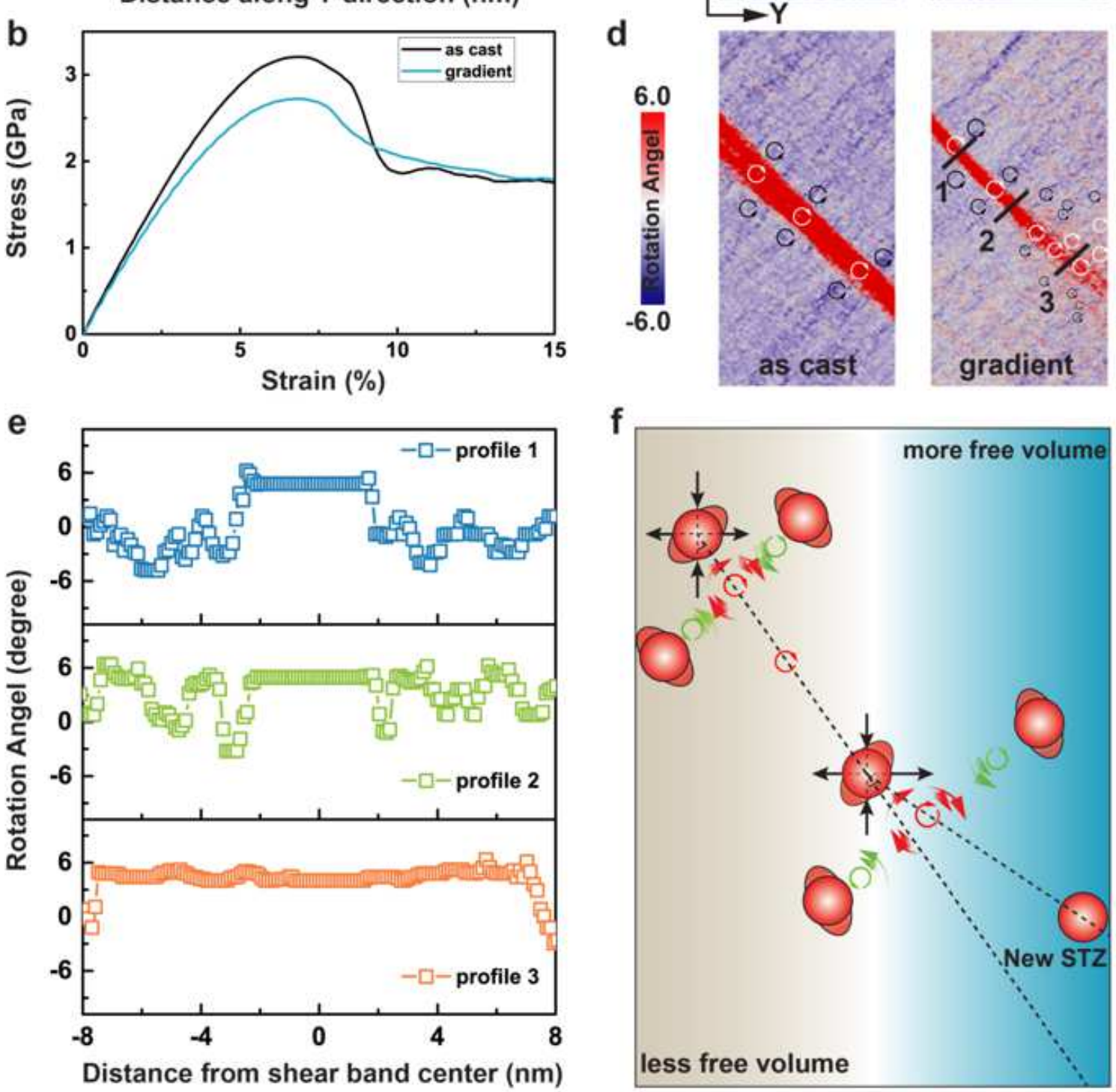

Figure 4

Investigation of the deformation behavior of GMG by MD simulations. (a) Atomic free volumes as the functions of the position along the Y-direction in as-cast and gradient MGs. Inset shows an illustration of the design of GMG. (b) Representative stress-strain results for the as-cast and gradient MGs during compression along the X-direction. (c) The spatial distribution of atomic Mises strain (d) Rotation angle 
of the as-cast and gradient MGs at $9 \%$ compression strain. (e) The variations of the rotation angle along lines 1-3 in (d). (f) Schematic illustration of the STZ percolation mechanism in GMG. 